\title{
Are midge swarms bound together by an effective velocity-dependent gravity?
}

\author{
Andrew M. Reynolds ${ }^{1, \text { a }}$, Michael Sinhuber ${ }^{2}$, and Nicholas T. Ouellette ${ }^{2}$ \\ 1 Rothamsted Research, Harpenden, AL5 2JQ, UK \\ 2 Department of Civil and Environmental Engineering, Stanford University, Stanford, CA 94305, USA
}

Received 21 December 2016 and Received in final form 1 March 2017

Published online: 14 April 2017 - C EDP Sciences / Società Italiana di Fisica / Springer-Verlag 2017

\begin{abstract}
Midge swarms are a canonical example of collective animal behaviour where local interactions do not clearly play a major role and yet the animals display group-level cohesion. The midges appear somewhat paradoxically to be tightly bound to the swarm whilst at the same time weakly coupled inside it. The microscopic origins of this behaviour have remained elusive. Models based on Newtonian gravity do, however, agree well with experimental observations of laboratory swarms. They are biologically plausible since gravitational interactions have similitude with long-range acoustic and visual interactions, and they correctly predict that individual attraction to the swarm centre increases linearly with distance from the swarm centre. Here we show that the observed kinematics implies that this attraction also increases with an individual's flight speed. We find clear evidence for such an attractive force in experimental data.
\end{abstract}

Collective behaviour of groups of social animals is ubiquitous and occurs across taxa, and because of this has a long modelling history [1]. These models strive to tease out the general dynamics that underlie the emergence of collective movements. Mating swarms of flying insects are particularly challenging in this regard because in contrast with fish schools and bird flocks, local interactions appear not to play a key role and yet the animals display group-level cohesion [2-4]. Here we construct dynamical models that are consistent with density profiles and velocity statistics measured from high-resolution, high-speed video recordings of swarms of the non-biting midge Chironomus riparius [5]. We show that these models predict the occurrence of velocity-dependent restoring forces and we uncover evidence for such interactions in existing experimental data.

Antecedents of our claims can be found in the groundbreaking studies of Okubo [6] and in the recent work of Gorbonos et al. [7]. Okubo [6] speculated that midge swarms are analogous to self-gravitating systems and, as a result, individuals are attracted to the centre of the swarm by an effective (fictitious) net force that increases linearly with distance from the swarm centre, as would be the case if the gravity were Newtonian and if the midges were uniformly distributed in space [8]. There is strong experimental support for such a net linear restoring force [5]. Okubo [6] did, however, recognise that midge density profiles are nearly Gaussian rather than uniform and this re-

\footnotetext{
a e-mail: andy.reynolds@rothamsted.ac.uk (corresponding
} author) quires that gravity be "adaptive" rather than purely Newtonian, as first noted by Gorbonos et al. [7]. Gorbonos et al. [7] suggested that midges interact primarily via longrange acoustic sensing. They subsequently exploited the similarity in form between the decay of acoustic and gravitational sources to build a model of swarm behaviour. Acoustic perception typically adapts to the overall sound level. This adaptive response renormalizes the effective forces according to the local noise amplitude. This is a common feature of biological sensory organs, preventing damage and their saturation. The model of Gorbonos et al. [7] is motivated by the fact that midges are expected to be very sensitive to acoustic signals. The modelled kinematics (swarm density profiles, velocity and acceleration statistics) were broadly consistent with observed density profiles and with observed distributions of velocity and acceleration.

In contrast to the approach taken by Gorbonos et al. [7] we do not posit a microscopic model and then test for compliance with observations but instead devise a model that by construction is necessarily consistent with the observed kinematics. We assume that the positions, $\boldsymbol{x}$, and velocities, $\boldsymbol{u}$, of individual midges can be described by the stochastic differential equations

$$
\begin{aligned}
\mathrm{d} u_{i} & =a_{i}(u, x, t) \mathrm{d} t+b(u, x, t) \mathrm{d} W_{i}(t), \\
\mathrm{d} x_{i} & =u_{i} \mathrm{~d} t,
\end{aligned}
$$

where the subscripts denote Cartesian components and where $\mathrm{d} W(t)$ is an incremental Wiener process with correlation property $\overline{\mathrm{d} W_{i}(t) \mathrm{d} W_{j}(t+\tau)}=\delta(\tau) \delta_{i j} \mathrm{~d} t$. The noise 
a)

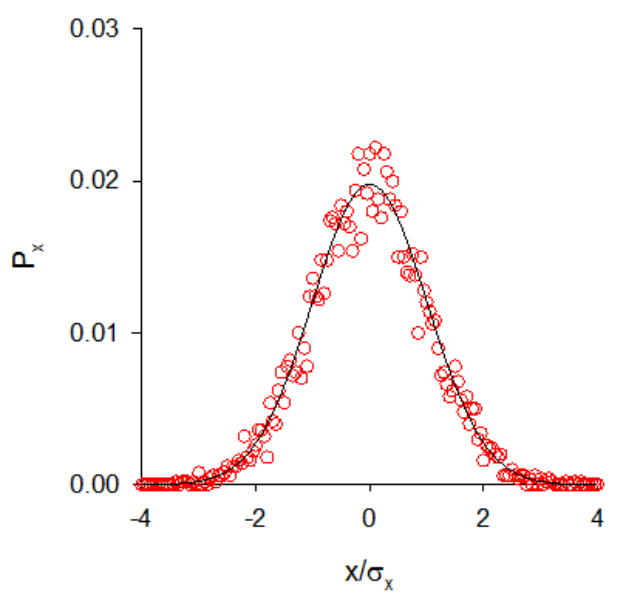

c)

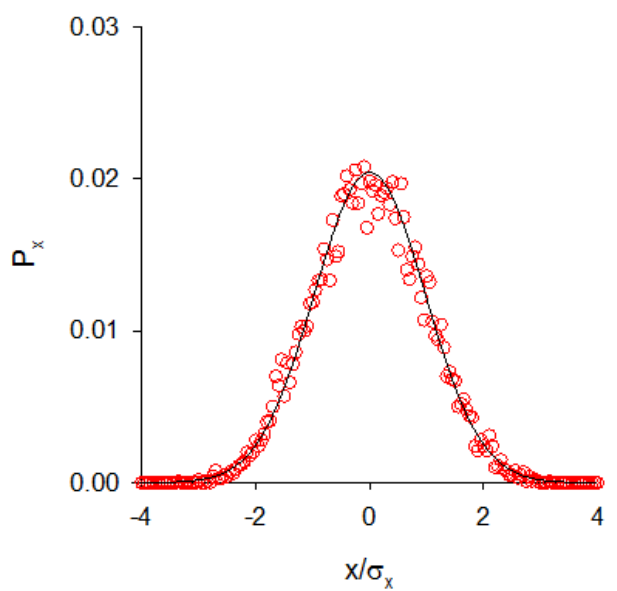

b)

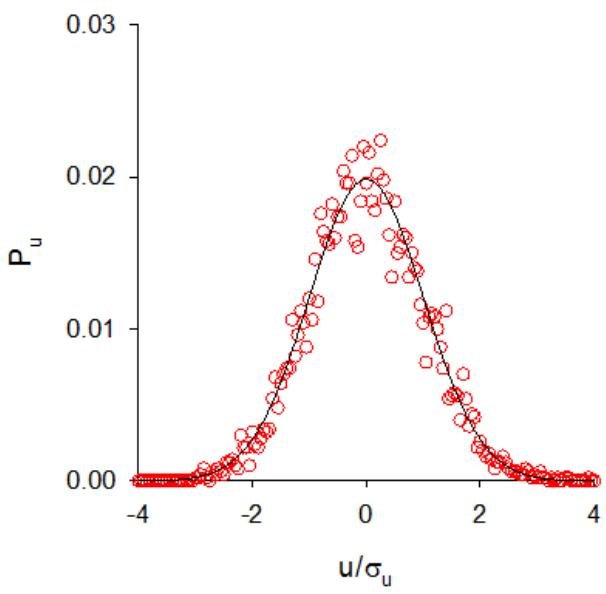

d)

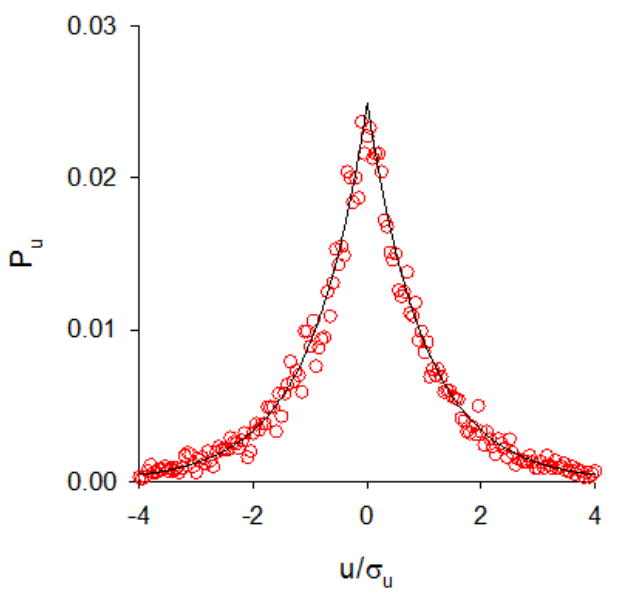

Fig. 1. Simulation data (o) for swarm density profiles and swarm velocities showing consistency prescribed statistics (solid lines) used as model inputs. Data are shown for the Gaussian velocity model (eq. (7)) (upper panels) and the exponential velocity model (eq. (8)) (lower panels).

term, $b \mathrm{~d} W_{i}$, models a stochastic component of the resultant internal forces that arise because of the limited number of individuals within the swarm, the non-uniformity of their spatial distribution, chance encounters with other individuals, and perhaps because of the inherent uncertainties in the detection of conspecifics. Stochasticity is a crucial aspect of midge dynamics but is not significant in most celestial systems [9], "collisional systems" being an exception. We further assume that the magnitude of the driving noise, $b(u, x, t)$, is a constant. Equation (1) is effectively a first-order autoregressive stochastic process in which position and velocity are modelled as a joint Markovian process. At second order, position, velocity and acceleration would be modelled collectively as a Markovian process. Physically, this hierarchy of models corresponds to the inclusion of a velocity autocorrelation timescale at first order, the addition of an acceleration autocorrelation timescale at second order, and so on [10]. We do not account explicitly for interactions between individuals. This is reasonable because measurements of the mean free path of the insects suggest that individuals are on average very weakly coupled, and yet tightly bound to the swarm itself $[2,11]$. Moreover, model predictions for average net forces do not change when the order of the modelling is increased [12].

The deterministic term, $a(u, x, t)$, is here determined by the requirement that the distributions of modelled positions and velocities be consistent with the observed forms of the midge density profile and velocity distributions. Mathematically, these consistency conditions require that $a_{i}(u, x, t)$ be a solution of the Fokker-Planck equation

$$
\frac{\partial P}{\partial t}+u_{i} \frac{\partial P}{\partial x_{i}}=-\frac{\partial}{\partial u_{i}}\left(a_{i} P\right)+\frac{b^{2}}{2} \frac{\partial^{2} P}{\partial u_{i}^{2}},
$$

where $P(u, x, t)$ is the joint distribution of velocity and position [13]. For statistically stationary swarms (with $\partial P(\partial t=0)$, the solution to eq. (2) for $a_{i}(u, x)$ can be 
written as

$$
a_{i}=\frac{b^{2}}{2} \frac{\partial \ln P}{\partial u_{i}}+\frac{\phi_{i}}{P},
$$

where the function $\phi_{i}$ is determined by

$$
\frac{\partial \phi_{i}}{\partial u_{i}}=-u_{i} \frac{\partial P}{\partial x_{i}} .
$$

The first term on the right-hand side of eq. (3) is a memory or relaxation term that drives velocities back towards their equilibrium values. The second term is effectively the conditional mean acceleration, $\langle A \mid u, x\rangle$.

In contrast with bird flocks, laboratory insect swarms are fairly similar in all three dimensions and are only weakly axisymmetric [5]. We therefore assume here that the swarms are spherically symmetric. In this case eq. (4) is most naturally expressed in spherical coordinates as

$$
\begin{aligned}
& \frac{1}{s^{2}} \frac{\partial}{\partial s}\left(s^{2} \phi_{s}\right)+\frac{1}{s \sin \hat{\varphi}} \frac{\partial}{\partial \hat{\varphi}}\left(\phi_{\hat{\varphi}} \sin \hat{\varphi}\right)+\frac{1}{s \sin \hat{\varphi}} \frac{\partial \phi_{\hat{\theta}}}{\partial \hat{\theta}}= \\
& -s[\sin \hat{\varphi} \sin \varphi \cos (\hat{\theta}-\theta)+\cos \hat{\varphi} \cos \varphi] \frac{\partial P}{\partial r},
\end{aligned}
$$

where $x_{1}=r \cos \theta \sin \varphi, x_{2}=r \sin \theta \sin \varphi, x_{3}=r \cos \varphi$, $u_{1}=s \cos \hat{\theta} \sin \hat{\varphi}, u_{2}=s \sin \hat{\theta} \sin \hat{\varphi}, u_{3}=s \cos \hat{\varphi}, r$ is the radial distance from the swarm centre and $s$ is the midges' flight speed. For swarms with Gaussian density profiles, $\frac{\partial P}{\partial r}=-\frac{r}{\sigma_{r}^{2}} P$, where $\sigma_{r}$ is the root-mean-square size of the swarm. A simple solution to eq. (5) for the conditional mean accelerations is

$$
\begin{aligned}
& \phi_{s}=-\frac{r}{\sigma_{r}^{2}}[\sin \hat{\varphi} \sin \varphi \cos (\hat{\theta}-\theta)+\cos \hat{\varphi} \cos \varphi] \frac{1}{s^{2}} \int_{s}^{\infty} s^{3} P \mathrm{~d} s, \\
& \phi_{\hat{\theta}}=0, \\
& \phi_{\hat{\varphi}}=0 .
\end{aligned}
$$

In these models conditional mean accelerations (i.e., the effective net restoring forces) increase linearly with distance from the swarm centroid, in agreement with observations, and mirroring the models of Okubo [6] and Gorbonos et al. [7]. Moreover, simulated midges tend to travel back and forth through the swarm centroid, in accordance with observations [3]. By way of contrast, models with $\phi_{\hat{\theta}} \neq 0$ are not self-consistent because simulated swarms cannot be constrained in the $z$-direction and models with $\phi_{\hat{\varphi}} \neq 0$ predict that midges have a tendency to orbit around the swarm centroid. Non-Gaussian density profiles are associated with modelled conditional mean accelerations that vary non-linearly with distance from the swarm centroid, i.e., with non-Newtonian gravitationallike forces.

For Gaussian velocities, the mean accelerations (restoring forces)

$$
\frac{\phi_{s}}{P}=-3 r \frac{\sigma_{u}^{2}}{\sigma_{r}^{2}}[\sin \hat{\varphi} \sin \varphi \cos (\hat{\theta}-\theta)+\cos \hat{\varphi} \cos \varphi]
$$

do not depend on velocity and so mirror the modelling of Okubo [6]. However, any departure from Gaussian velocity statistics leads to velocity-dependent restoring forces.

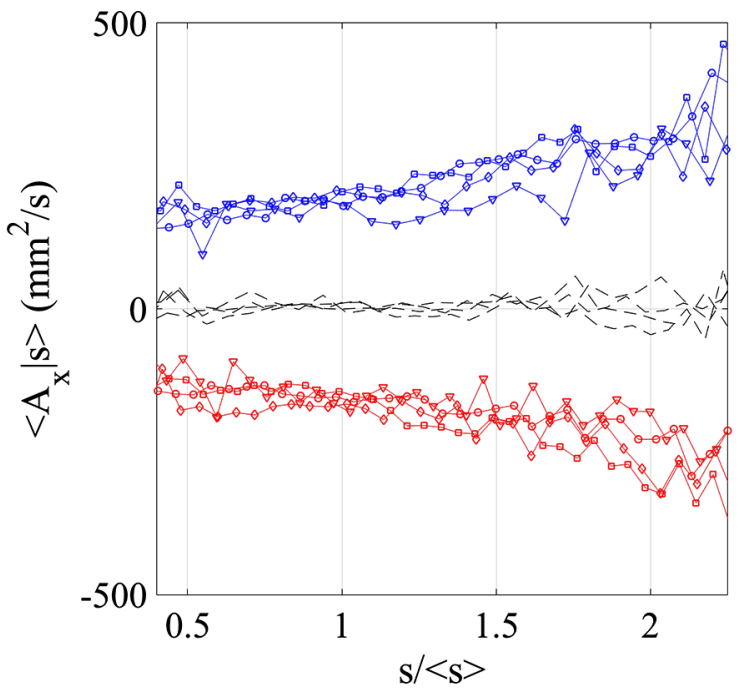

Fig. 2. Empirical data for the mean value of a single component (in the radial plane) of the acceleration $A_{x}$ conditioned on midge speed $s$, computed from the data set described in [14]. Data are shown for groups of similarly sized swarms (6 to 15 insects (o); 16 to $25(\square) ; 26$ to $35(\diamond) ; 36$ to $45(\triangle))$ and averaged over the central region of each swarm (out to $1 / 2$ of the swarm radius). Blue symbols show data only for the left hemisphere, and red symbols only for the right hemisphere; the dashed lines show data for both hemispheres, which (as required by symmetry) is close to zero. For the single-hemisphere data, the conditional acceleration increases as the speed increases, in agreement with eq. (8).

This arises, for instance, when the Gaussian velocity distribution is truncated to account for the fact that midges have a maximum flight speed. It also arises when, in accordance with observations [5], velocities are taken to be non-Gaussian. If, for example, speeds are exponentially distributed with mean $\bar{s}$, then

$$
\frac{\phi_{s}}{P}=-2 r \frac{\bar{s}}{\sigma_{r}^{2}}[\sin \hat{\varphi} \sin \varphi \cos (\hat{\theta}-\theta)+\cos \hat{\varphi} \cos \varphi][\bar{s}+s],
$$

whose validity was confirmed by numerical integration (fig. 1). In this regard velocity-dependent restoring forces are the norm rather than the exception. In the former case of truncated Gaussian velocity distributions, the restoring forces weaken with increasing flight speed whilst in the case of exponential velocity distributions their strength increases with flight speed in accordance with our observations (fig. 2). Reynolds and Ouellette [12] recently reported on analogous results for a 1-dimensional model, but such models cannot be directly construed as gravity models because the effective forces depend on the distance to a principal axis rather than on the distance to the swarm centre.

It is readily shown that modelled conditional acceleration cannot increase linearly with distance from the swarm centroid if the velocity distribution is position dependent and when, as observed, density profiles are Gaussian or are close to Gaussian. Therefore, we did not consider such models but note that such distributions can re- 


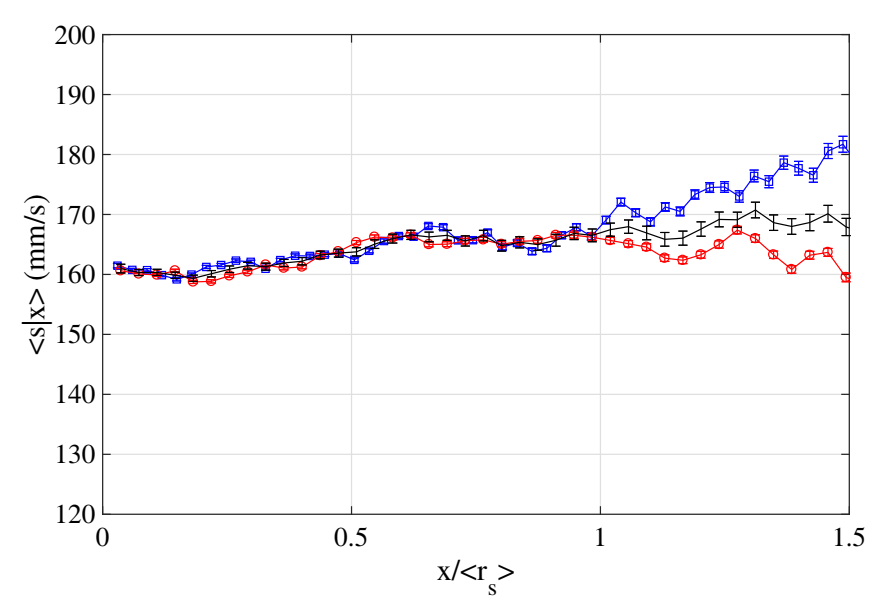

Fig. 3. Empirical data for the speed (in the radial plane) conditioned on position normalized by the swarm size $\left\langle r_{s}\right\rangle$, computed from the data set described in [14]. The blue line shows data only for the left hemisphere, and the red line only for the right hemisphere; the black line shows data for both hemispheres.

sult in correlations between net forces and velocities and could thereby give the impression of velocity-dependent restoring forces. There is however scant empirical evidence for velocity statistics depending on position (fig. 3). Velocities might conceivably have decreased with distance from the swarm centroid but in this case, net forces would then appear to decrease with speed rather than increase with speed, counter to our observations (fig. 2).

In this letter we have built upon the pioneering work of Okubo [6] and on the recent advances made by Gorbonos et al. [7], who suggested that midge swarms are analogous to self-gravitating systems. These models predict correctly that midges are effectively bound to the swarm centre by a restoring force that increases linearly with distance from the swarm centre. Here we showed both theoretically and empirically that this restoring force also depends on speed. This insight may lead to a more detailed understanding of the origins of interactions between midges. It is possible that velocity-dependent restoring forces of the particular kind observed in experiments can be recovered from adaptive Newtonian gravity models. If not, then interactions between midges may be speed dependent. This is not implausible from a biological perspective. As asserted above, the gravity-like interactions that bind midge swarms together probably arise from acoustic sensing. Midges detect sound by transducing the mechanical signal from the bending of hairs on their antennae. Thus, they are sensitive to the actual air flow induced by acoustic waves [15]. And behaviourally, midges tend to fly fastest and to execute their most intense flight manoeuvres when either chasing other midges or being chased themselves; thus, these behavioural mechanisms could also be responsible for our observations. Another possibility is that the long-range midge interactions may also be partially visually cued, as they are in bird flocks [16]. Solid angles decrease as inverse-square power laws and it is well known that animals are often easiest to detect when in motion [17]. Although the detailed biophysical explanation for the swarm dynamics we observe warrants further examination, our analysis does self-consistently show that the net effect of the underlying mechanisms produces a speed-dependent force that effectively binds midges to the centre of a swarm.

If interactions between midges were found to depend on speed, then midge swarms would be bound together by an effective velocity-dependent gravity. This brings to mind Gerber's $[18,19]$ long-forgotten theory of speeddependent gravity. The theory was quickly rejected by the astronomical community after being met with fierce criticism because the theoretical derivation is incoherent and because its predictions conflict with astronomical observations. Our analysis suggests that Gerber's idea of a velocity-dependent gravity (though not his derivation and reasoning) might be resurrected in this different context, as midge swarms could effectively behave as selfgravitating systems bound together by speed-dependent forces.

Rothamsted Research receives grant aided support from the Biotechnology and Biological Sciences Research Council. MS and NTO acknowledge support from the U.S. Army Research Office under grant No. W911NF-16-1-0185. We thank Nir Gov and Dan Gorbonos for many insightful comments.

\section{Author contribution statement}

AMR and NTO devised the research. AMR undertook the theoretical analysis. MS analysed the experimental data. AMR, MS and NTO wrote the paper.

\section{References}

1. D.J.T. Sumpter, Collective Animal Behavior (Princeton University Press, 2010).

2. J.G. Puckett, D.H. Kelley, N.T. Ouellette, Sci. Rep. 4, 4766 (2014)

3. R. Ni, J.G. Puckett, E.R. Dufresne, N.T. Ouellette, Phys. Rev. Lett. 115, 118104 (2015).

4. R. Ni, N.T. Ouellette, Phys. Biol. 13, 045002 (2016).

5. D.H. Kelley, N.T. Ouellette, Sci. Rep. 3, 1073 (2013).

6. A. Okubo, Adv. Biophys. 22, 1 (1986).

7. D. Gorbonos, R. Ianconescu, J.G. Puckett, R. Ni, N.T. Ouellette, N.S. Gov, New J. Phys. 18, 073042 (2016).

8. A.S. Ramsey, Newtonian Attraction (Cambridge University Press, 1981).

9. S. Chandrasekhar, Rev. Mod. Phys. 15, 1 (1943).

10. B.L. Sawford, Phys. Fluids A 3, 1577 (1991).

11. R. Ni, N.T. Ouellette, Eur. Phys. J. ST 224, 3271 (2015).

12. A.M. Reynolds, N.T. Ouellette, Sci. Rep. 6, 30515 (2016).

13. D.J. Thomson, J. Fluid Mech. 180, 529 (1987).

14. J.G. Puckett, N.T. Ouellette, J. R. Soc. Interface 11 , 20140710 (2014).

15. B. Bathellier, T. Steinmann, F.G. Barth, J. Casas, J. R. Soc. Interface 9, 1131 (2012).

16. D.J.G. Pearce, A.M. Miller, G. Rowlands, M.S. Turner, Proc. Natl. Acad. Sci. U.S.A. 111, 10422 (2014).

17. J.P. Hailman, Optimal Signals: Animal Communication and Light (Indiana University Press, 1977).

18. P. Gerber, Z. Math. Phys. 43, 93 (1898).

19. P. Gerber, Ann. Phys. (Berlin) 52, 415 (1902). 